\title{
New Editorial Hypertension Research: Looking back to 2021 and perspective to 2022
}

\author{
Kazuomi Kario ${ }^{1} \cdot$ Masaki Mogi $^{2} \cdot$ Satoshi Hoshide ${ }^{1}$
}

Received: 22 November 2021 / Accepted: 22 November 2021 / Published online: 28 December 2021

(c) The Japanese Society of Hypertension 2021

The official journal of the Japanese Society of Hypertension, "Japanese Journal of Hypertension", was first published in April 1978. Fourteen years later, in April 1992, it was revised to the English journal "Hypertension Research", which is the official journal of the Japanese Society of Hypertension (Fig. 1). We have 24 national Associated Editors, 16 international Associated Editors, and over 350 reviewers helped with the peer review process. The peer review and publication process were much shorter than in the past, and the number of submissions exceeds 700 per year, with the impact factor rising to 3.872 in 2021 and keep the stable acceptance rate in recent years (Fig. 2).

In 2021, we have simultaneously published 9 papers of the 20 late-breaker abstracts presented at the 43rd Annual
Scientific Meeting of the Japanese Society of Hypertension in Okinawa, and all papers appear in January and February issues. We express deep appreciation to Prof. Yusuke Ohya, the President, and Dr. Atsushi Sakima, the Secretary-General of Okinawa meeting for their generous full support to proceed with the rapid review process and publish all papers promptly. We have also released Okinawa declaration on the diversity for the management of hypertension with each Asian Society of Hypertension [1]. As a new activity, we are starting an annual report [2], in which experts in their fields will summarize the papers published in Hypertension Research during the year. There is also a growing number of commentaries on the published papers, which provide a diverse overview of other

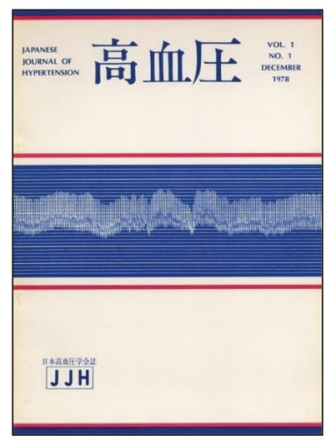

Apr. 1978

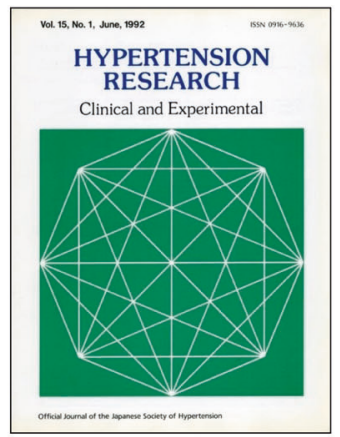

Jun. 1992

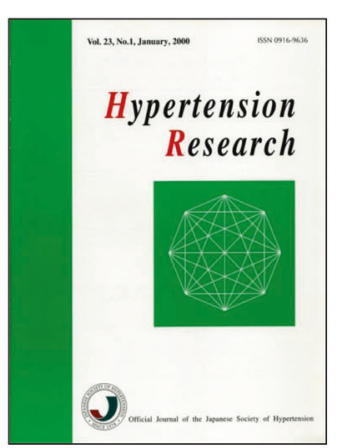

Jan. 2000

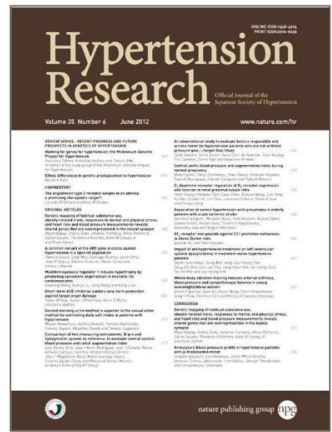

Jan. 2009

Fig. 1 Chronicle of the hypertension research

Kazuomi Kario

kkario@jichi.ac.jp

1 Department of Cardiology, Jichi University School of Medicine, Tochigi, Japan
2 Department of Pharmacology, Ehime University, Graduate School of Medicine, Ehime, Japan 


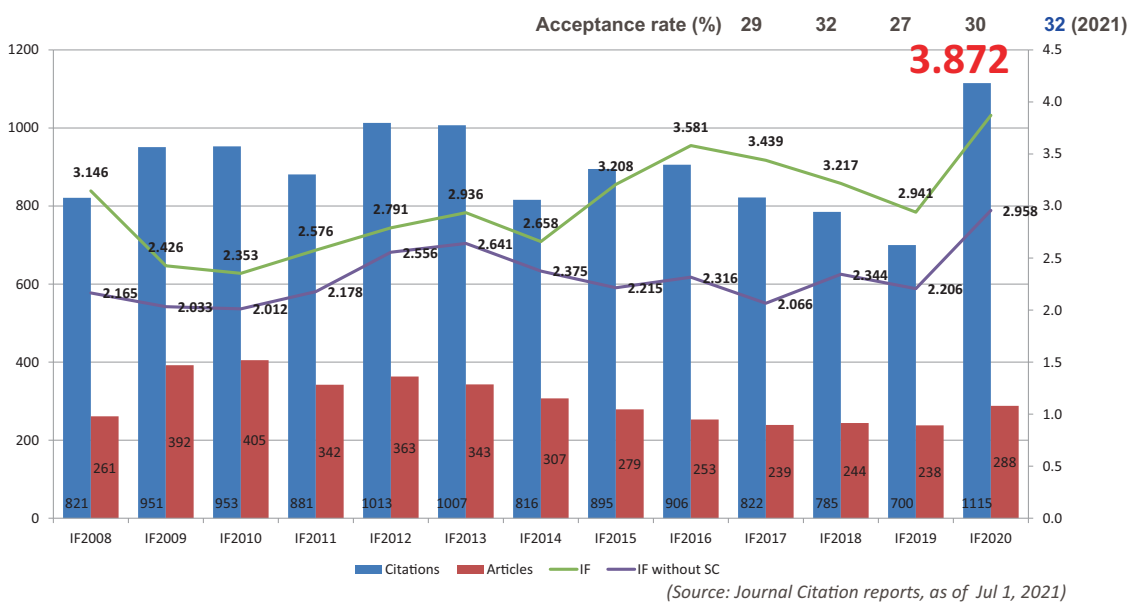

Fig. 2 Impact factors and acceptance rate in recent years

researchers in the field. The development of medical science begins with the recording of facts, which is the essential meaning of a medical journal. Since the end of last year, we have expanded the scope of submissions. It now includes organ damage, vascular dysfunction, cerebrovascular disease, chronic kidney disease, metabolic diseases, and important issues in specific populations such as the elderly (cognitive dysfunction, frailty), children, pregnant women, cancer patients etc. Moreover, papers on outcome research concerning hypertension and its management and the use of emerging technologies such as telemedicine, big data analysis, wearable devices, artificial intelligence (AI), and other digital technologies are also welcome. Further, we added the new content, "Brief communication" and "Literature Review and Cases with Typical Imaging (Clinical or Experimental)". For more information, please see Guide to Authors. As an editorial team, we would like you to consider Hypertension Research as a stance to record new perspectives that you consider important and to make an academic contribution to society through your expertise. In 2022, we will use social networking services more than ever to disseminate information about Hypertension Research.

In addition, the 29th Scientific Meeting of the International Society of Hypertension (Hypertension Kyoto 2022) will be held in Japan this year, which will be a very exciting year for the Japanese Society of Hypertension. With the advantage of being an English-language journal with more than 40 years of history in Japan, we would like to (1) further internationalize the journal, (2) develop it into a stance for real-time information transmission and exchange of the Japanese Society of Hypertension to the world, and (3) make it a gateway for young researchers. We would like to ask for your continued support for Hypertension Research.

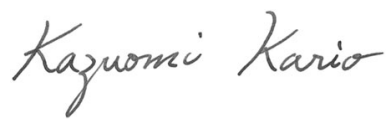

Kazuomi Kario, Editor-in-Chief

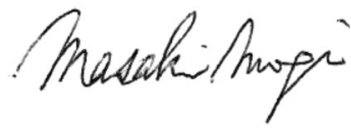

Masaki Mogi, Deputy Editor

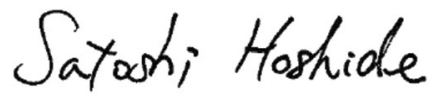

Satoshi Hoshide, Managing Editor

Acknowledgements The authors gratefully acknowledge the full support of editorial assistant, Ms. Kyoko Takayama at the Japanese Society of Hypertension and the publishing team, Ms. Sachiko Hayakawa, Ms. Mariko Kubota, and Ms. Akemi Yoshida at Springer Nature.

Publisher's note Springer Nature remains neutral with regard to jurisdictional claims in published maps and institutional affiliations. 


\section{References}

1. Ohya Y, Kario K, Itoh H, Nishiyama A, Ishimitsu T, Ichihara A, et al. Statement of the Asian Hypertension Society Network: the Okinawa Declaration on the unity of hypertension societies in Asian countries and regions to overcome hypertension and hypertension-related diseases. Hypertens Res. 2021. https://doi.org/10.1038/s41440-02100781-4

2. Mogi M, Higashi Y, Bokuda K, Ichihara A, Nagata D, Tanaka A, et al. Annual reports on hypertension research 2020. Hypertens Res. 2021. https://doi.org/10.1038/s41440-021-00766-3

\section{About Editors}

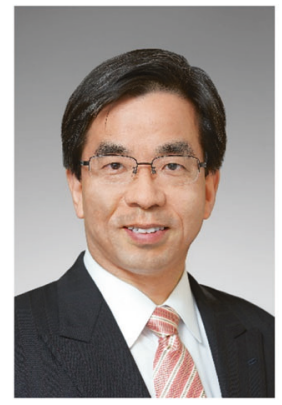

Editor-in-Chief

Kazuomi KARIO (Tochigi, Japan)

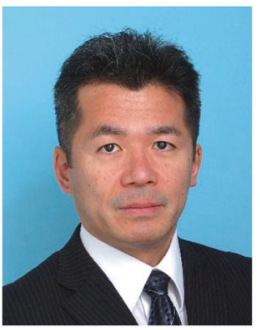

Deputy Editor Masaki MOGI (Ehime, Japan)

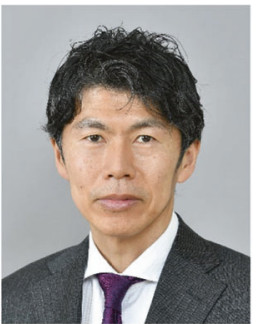

Managing Editor Satoshi HOSHIDE (Tochigi, Japan)

\section{- Social Media Section Editors}

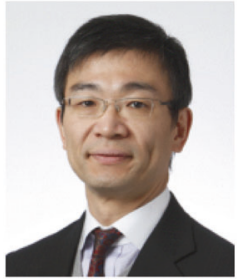

Tatsuo SHIMOSAWA

(Tokyo, Japan)

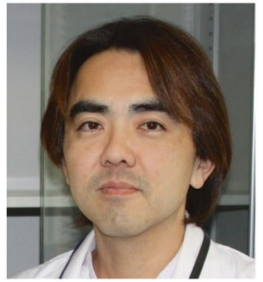

Takuya KISHI (Fukuoka, Japan) 
- Consulting Editors

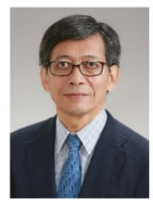

Toshihiko

Toshihiko
ISHIMITSU (Tochigi, Japan)

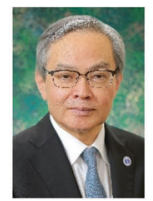

Naoki

KASHIHARA (Okayama, Japan)

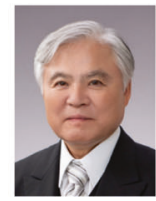

Sadayoshi ITO (Miyagi, Japan)

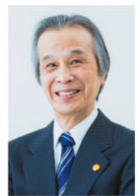

Kazuaki SHIMAMOTO (Hokkaido, Japan)

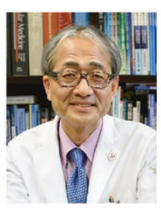

Hiroshi ITOH (Tokyo, Japan)

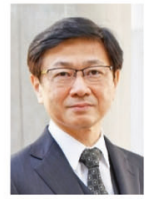

Hirom

RAKUG

(Osaka, Japan)

\section{- International Consulting Editors}

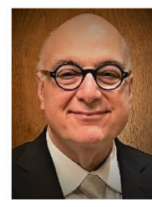

George L. BAKRIS (Chicago, USA)

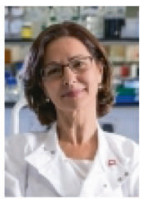

Rhian TOUYZ (Glasgow, UK)

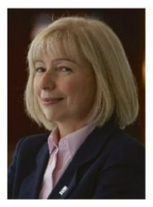

Anna F. DOMINICZAK (Glasgow, UK)

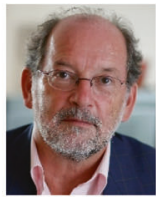

Thomas UNGER (Maastricht, The Netherlands

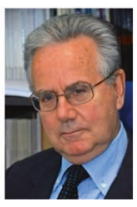

Giuseppe MANCIA (Monza, Italy)

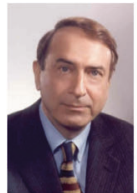

Michael WEBER (New York, USA)

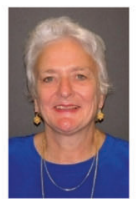

Suzanne OPARIL (Alabama, USA)

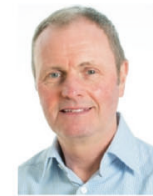

Bryan WILLIAM (Leicester, UK)

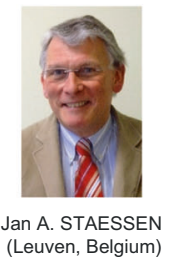

(LeUven, Belgium)

\section{- Associate Editors}

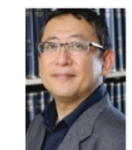

Hisatomi ARIMA (Fukuoka, Japan)

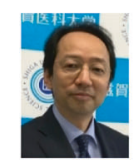

Katsuyuki MIURA

(Shiga, Japan)

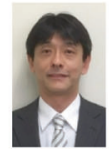

Takayoshi OHKUBO (Tokyo, Japan)

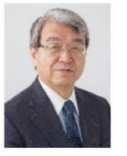

Naoyuki

HASEBE

(Hokkaido, Japan) (Hiroshima, Japan)

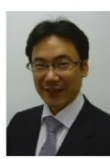

Takefumi MORI (Miyagi, Japan)

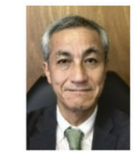

Yusuke OHYA (Okinawa, Japan) (Tokushima,
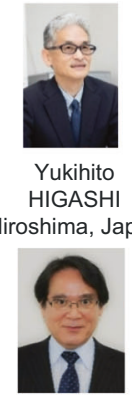

Masashi (Kumamoto, Japan)

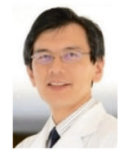

Tokushima, Japan)

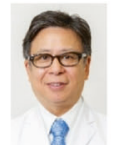

Atsuhiro ICHIHARA (Tokyo, Japan)

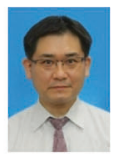

Daisuke NAGATA (Tochigi, Japan)

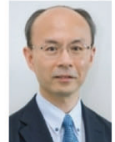

Hirotaka SHIBATA (Oita, Japan)

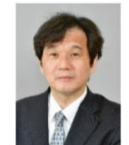

Yasusi IMAI (Tochigi, Japan)

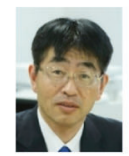

Hironori NAKAGAMI (Osaka, Japan)

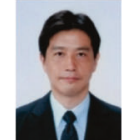

Shigeru SHIBATA (Tokyo, Japan)

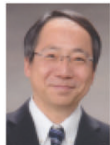

Hisashi KAl (Fukuoka, Japan)

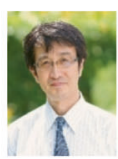

Akira NISHIYAMA (Kagawa, Japan)

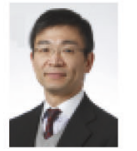

Tatsuo SHIMOSAWA (Tokyo, Japan)

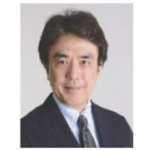

Norihiro KATO (Tokyo, Japan)

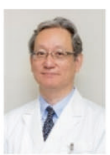

Koichi NODE (Saga, Japan)

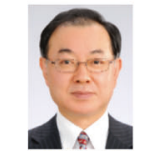

Koichi TAMURA (Yokohama, Japan)
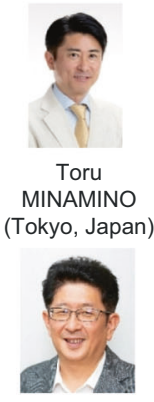

Mitsuru OHISHI (Kagoshima, Japan)

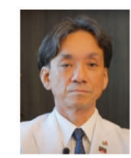

Kazunori TOYODA (Osaka, Japan) 


\section{- International Associate Editors}

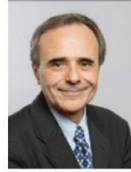

Michel

AZIZI

(Paris, France)

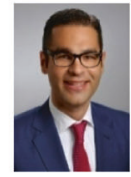

Felix MAHFOUD (Homburg, Germany)

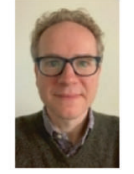

James Brian

BYRD

(Michigan, USA)

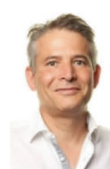

Richard McMANUS (London, UK)

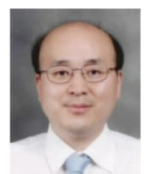

Sungha PARK (Seoul, South Korea)

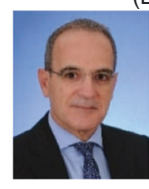

George S.

STERGIOU

(Athens, Greece)

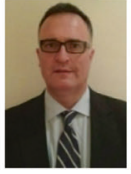

Miguel

CAMAFORT

(Barcelona, Spain)

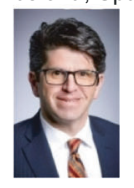

Paul MUNTNER

(Alabama, USA)

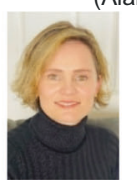

Alta SCHUTTE (Sydney, Australia)

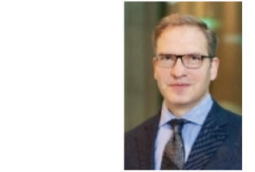

Jens JORDAN (Cologne, Germany)

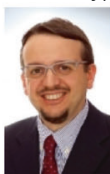

Stefano OMBONI (Varese, Italy)

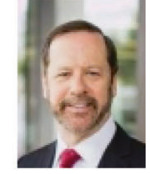

Theodore W. KURTZ

(San Francisco, USA)

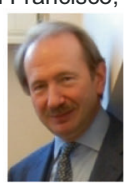

Gianfranco PARATI

(Milan, Italy)

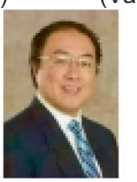

Daichi SHIMBO (New York, USA)

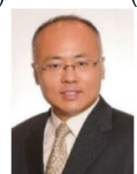

Ji-Guang WANG

(Shanghai, China)

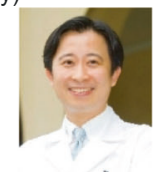

Tzung-Dau WANG

(Taipei, Taiwan) 